\title{
Targeting ROCK2 rather than ROCK1 inhibits Ewing sarcoma malignancy
}

\author{
ROSA SIMONA PINCA ${ }^{1}$, MARIA CRISTINA MANARA ${ }^{1}$, VALENTINA CHIADINI ${ }^{1}$, \\ PIERO PICCI $^{1}$, CINZIA ZUCCHINI ${ }^{2 *}$ and KATIA SCOTLANDI ${ }^{1 *}$ \\ ${ }^{1}$ CRS Development of Biomolecular Therapies, Experimental Oncology Laboratory, \\ Rizzoli Orthopedic Institute, I-40136 Bologna; ${ }^{2}$ Department of Experimental, \\ Diagnostic and Specialty Medicine, University of Bologna, I-40126 Bologna, Italy
}

Received May 19, 2016; Accepted June 29, 2016

DOI: $10.3892 /$ or.2017.5397

\begin{abstract}
Understanding the molecular processes characterizing Ewing sarcoma (EWS) cell migration is crucial to highlight novel therapies for patients with disseminated disease. In this study we analyzed the role of ROCK kinases in the regulation of cell migration, growth and differentiation of EWS cells. Overexpression of ROCK promotes invasion and metastasis in many solid tumors. However, the effect of ROCK in EWS has not been extensively investigated. Expression of ROCK1 and ROCK2 was analyzed by western blotting in a representative panel of human EWS cell lines, in comparison with the parameters of in vitro malignancy. We investigated the effects of a ROCK2 specific inhibitor toward those of a pan-ROCK inhibitor on the growth, migration and differentiation of two EWS cell lines. ROCK2 but not ROCK1 expression was found to be associated with in vitro cell migration and anchorage-independent growth capabilities. Exposure of EWS cells to ROCK inhibitors significantly reduced migration and growth, while favoring morphology changes and neural differentiation. These effects were more striking when cells were specifically deprived of ROCK 2 activity. Our findings lead to consider ROCK2, rather than ROCK1, as a possible molecular target for the treatment of EWS.
\end{abstract}

\section{Introduction}

Ewing sarcoma (EWS) is a malignant primitive bone tumor, that mainly affects children and young adults, with a high tendency to metastasize to lung and/or bone $(1,2)$. Unlike carcinomas, sarcomas arise abruptly and diffuse micrometastases are thought to be present at the time of diagnosis as indicated by

Correspondence to: Dr Katia Scotlandi, CRS Development of Biomolecular Therapies, Experimental Oncology Laboratory, Rizzoli Orthopedic Institute, via di Barbiano 1/10, I-40136 Bologna, Italy

E-mail: katia.scotlandi@ior.it

*Joint senior authorship

Key words: migration, differentiation, ROCK inhibition, Ewing sarcoma a survival rate as low as $5 \%$ in patients treated with surgery alone (3). Although significant improvements in prognosis have been reported in patients with localized tumors at diagnosis, prognosis for patients with metastasis remains very disappointing $(4,5)$ and less than few treatment options can be offered to metastatic patients, indicating that new drugs are required. Thus, the primary need in the field is a deeper understanding of the biology of the metastasic process in EWS in order to facilitate the development of therapeutic agents that may specifically counteract cell dissemination and optimize systemic-disease control.

The key cellular processes underlying metastasis include the ability of cancer cells to migrate toward new environments outside of the primary tumor. The two major types of cancer migration (mesenchymal or amoeboid) require different intracellular molecular signaling but both rely on driving forces generated by actin cytoskeleton dynamics. The actin status is in fact used as a signaling intermediate by several pathways, including the Rho/Rac GTPases and the Hippo-pathway, which culminate in the transcriptional regulation of cytoskeletal and growth-promoting genes, respectively. In particular, the main downstream effectors of the Rho family GTPases, ROCK1 and ROCK2, are serine-threonine kinases that, through the phosphorylation of several target substrates linked to cytoskeleton organization, control multiple events in cell migration, such as regulation of actomyosin contractility, formation of stress fibers, rear retraction and turnover of focal adhesions (6). The implication of ROCK1 and ROCK2 in cancer cell dissemination and metastasis is therefore not surprising. Although the effects of ROCK activity on migration and invasion have been found to be dependent on the cellular context, ROCK overexpression has been associated with greater invasion and poor survival in many tumors, including hepatocellular carcinoma (7), osteosarcoma (8), breast (9), testicular (10), colon (11) and bladder cancer (12). Conversely, ROCK inhibition or depletion frequently produces suppression of invasion and metastasis (7,13-15), thus supporting the view of ROCK inhibitors as a novel therapeutic strategy to block the migration and invasion of metastatic cancers. Most of the anti-ROCK agents currently available are inhibitors that simultaneously target both the two kinases ROCK1 and ROCK2, in the hypothesis that the two isoforms drive similar intracellular 
signaling pathways and biological processes. However, despite their high degree of sequence homology and substrate promiscuity, several studies have clearly reported how the two isoforms may exhibit individual physiological roles $(16,17)$ and give distinct contribution to cancer progression (18).

In this study, we analyzed the role of ROCK isoforms in EWS malignancy and evaluated the in vitro efficacy of pan- vs. specific ROCK inhibitors. Our results indicate that targeting of ROCK2 could represent an effective approach to counteract EWS malignancy in favor of cell differentiation.

\section{Materials and methods}

Cell lines and treatments. SK-ES-1, SK-N-MC, and RD-ES EWS cell lines were obtained from the American Type Culture Collection (ATCC; Manassas, VA, USA); TC-71 and 6647 cell lines were a generous gift from T.J. Triche (Children's Hospital, Los Angeles, CA, USA); WE-68 was established and kindly provided by F. van Valen (University Hospital Muenster, Muenster, Germany); A673, STA-ET 2.1 and STA-ET 2.2 EWS cell lines were a kind gift from $\mathrm{H}$. Kovar (St. Anna Kinderkrebsforshung, Vienna, Austria); the latter two cell lines were established from the primary tumor and a bone marrow infiltrate of the same patient (19). LAP-35 was previously established and characterized at the Istituto Ortopedico Rizzoli, Bologna, Italy (20). IOR/CAR was established and characterized at the Experimental Oncology Laboratory of the Istituto Ortopedico Rizzoli, Bologna, Italy, from an EWS patient. All cell lines were tested for the absence of mycoplasma contamination by MycoAlert ${ }^{\mathrm{TM}}$ Mycoplasma Detection kit (Lonza, Allendale, NJ, USA), last control March 2015, and authenticated by STR analysis using genRESVR MPX-2 and genRESVR MPX-3 kits (Serac, Bad Homburg, Germany). The following loci were verified: D16S539, D18S51, D19S433, D21S11, D2S1338, D3S1358, D5S818, D8S1179, FGA, SE33, TH01 and TPOX VWA. Last control was performed in November 2012. Cells were cultured in a humidified $5 \% \mathrm{CO}_{2}$ atmosphere at $37^{\circ} \mathrm{C}$ in Iscove Modified Dulbecco's medium (IMDM; Lonza) supplemented with $10 \%$ fetal bovine serum (FBS; EuroClone S.p.A, Milan, Italy), and $1 \%$ penicillin-streptomycin.

To inhibit ROCK kinases the pan-ROCK inhibitor $(R)-(+)-$ trans-4-(1-aminoethyl)-N-(4-pyridyl)cyclohexanecarboxamide dihydrochloride (Y27632) (Calbiochem, San Diego, CA, USA) as well as the ROCK2 inhibitor N-(2-(2-(dimethylamino) ethoxy)-4-(1H-pyrazol-4-yl)phenyl)-2,3dihydrobenzo[b][1,4] dioxine-2-carboxamide (Stemolecule ${ }^{\mathrm{TM}}$ ROCKII Inhibitor; Stemgent, Cambridge, MA, USA) were used.

Analysis of growth features in monolayer conditions. The effects of ROCK inhibition on cell growth was determined by daily harvesting of cells after the seeding of 25,000 cells $/ \mathrm{cm}^{2}$ (for the 6647 cell line) or 50,000 cells $/ \mathrm{cm}^{2}$ (for the SKES-1 cell line) in IMDM and $10 \%$ FBS. ROCK inhibitors $(10 \mu \mathrm{M})$ were added to the culture medium $24 \mathrm{~h}$ after seeding. Cell viability was determined by trypan blue vital cell count.

Motility assay. Motility assay was performed using Transwell chambers (Costar, Cambridge, MA, USA). Cells $\left(1 \times 10^{5}\right)$ were seeded in the upper compartment in the presence or not of the ROCK inhibitors $(10 \mu \mathrm{M})$, and incubated for $18 \mathrm{~h}$ in a humidified $5 \% \mathrm{CO}_{2}$ atmosphere at $37^{\circ} \mathrm{C}$. The migrated cells were fixed in absolute methanol, counterstained with Giemsa (CARLO ERBA Reagents S.A.S., Milan, Italy) and counted. All the experiments were performed in triplicate.

Soft agar assay. Anchorage-independent growth was determined in $0.33 \%$ agarose (SeaPlaque ${ }^{\mathrm{TM}}$ Agarose; Lonza) with a $0.5 \%$ agarose underlay. Cell suspensions $\left(1 \times 10^{3}\right)$ were plated in semisolid medium (IMDM 10\% FBS plus agar 0.33\%) in the presence or not of the ROCK inhibitors $(10 \mu \mathrm{M})$, and incubated at $37^{\circ} \mathrm{C}$ in a humidified $5 \% \mathrm{CO}_{2}$ atmosphere. Colonies were counted after 10 to 14 days. All of the experiments were performed in triplicate.

Western blotting. Cells were lysed with phospho-protein extraction buffer containing $50 \mathrm{mM}$ Tris-HCl, $\mathrm{pH} 7.4,150 \mathrm{mM} \mathrm{NaCl}$, $1 \%$ NP-40, 0.25\% Na-deoxycholate, 1 mM EGTA, 1 mM NaF, supplemented with protease-phosphatase cocktail inhibitor (Sigma-Aldrich, St. Louis, MO, USA). Equivalent amounts of total cell lysates were separated by $7.5 \%$ SDS-PAGE under denaturating conditions and transferred onto nitrocellulose membrane. Membranes were incubated overnight with the following primary antibodies: rabbit polyclonal anti-ROCK1 (H-85, sc-5560; Santa Cruz Biotechnology, Inc., San Diego, CA, USA) (1:1,000), goat polyclonal anti-ROCK2 (C-20, sc-1851; Santa Cruz Biotechnology) (1:1,000), mouse monoclonal antiactin (C-4, MAB1501; Chemicon International, Inc., Temecula, CA, USA) $(1: 100,000)$. Donkey anti-rabbit (NA934; GE Healthcare, Piscataway, NJ, USA), donkey anti-goat (sc-2020; Santa Cruz Biotechnology) or sheep anti-mouse (NA931; GE Healthcare) horseradish peroxidase-linked secondary antibodies were employed and the signal was revealed by ECL western blotting detection reagents (EuroClone). Densitometric analysis was performed using GS-800 Imaging Densitometer and Quantity One 4.6.9 software (Bio-Rad Laboratories, Inc., Hercules, CA, USA).

Immunofluorescence. EWS cells were seeded at low density on fibronectin-coated coverslips in standard medium. After $48 \mathrm{~h}$, cells were exposed to ROCK inhibitors $(2 \mu \mathrm{M}$ or $10 \mu \mathrm{M})$. Twenty-four hours later, the cells were fixed in $4 \%$ paraformaldehyde, permeabilized with $0.15 \%$ Triton $\mathrm{X}-100$ in phosphate-buffered saline (PBS), and incubated overnight with the mouse monoclonal anti- $\beta$ III-tubulin antibody (SDL.3D10, T5076; Sigma-Aldrich) (1:50). Goat polyclonal anti-mouse FITC (31569; Pierce Biotechnology, Inc., Rockford, IL, USA) (1:100) was used as a secondary antibody. Nuclei were counterstained with Hoechst 33256 (Sigma-Aldrich). For neurite outgrowth assay, the cells were classed as differentiated if they exhibited an outgrowth extending from the cell which was at least 1.5 times the diameter of the cell. At least 200 cells from five randomly selected fields were counted from each slide.

Analysis of apoptosis. Detection and quantification of apoptotic cells was obtained by flow cytometric analysis (FACSCalibur; Becton Dickinson, San Jose, CA, USA) of Annexin V-FITC-labeled cells. This test was carried out according to the manufacturer's instructions (code no. 4700, 
A

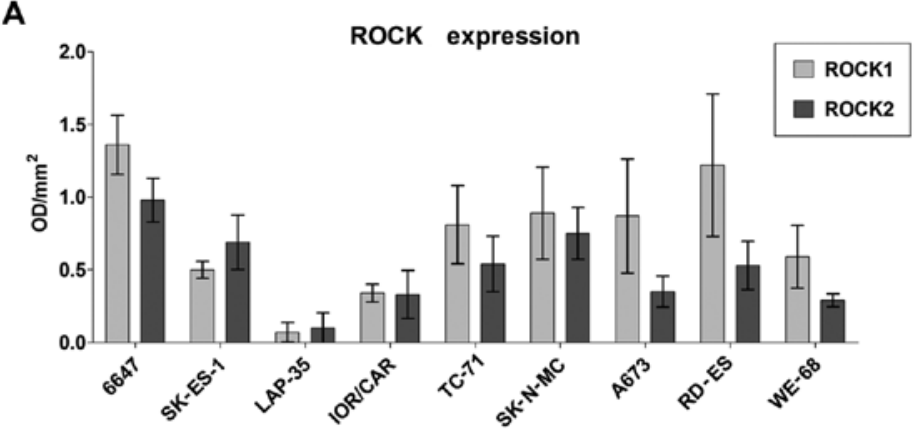

B

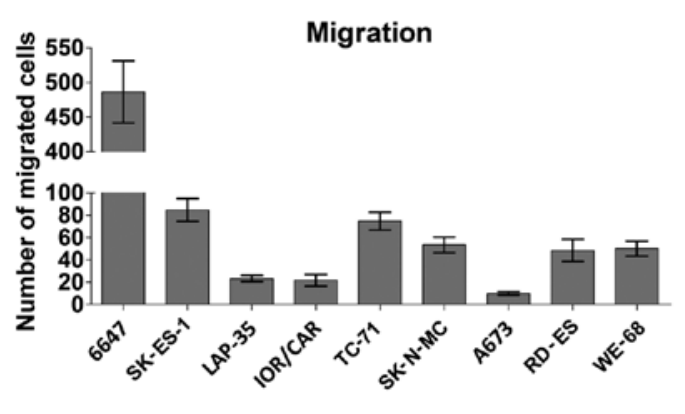

D



E

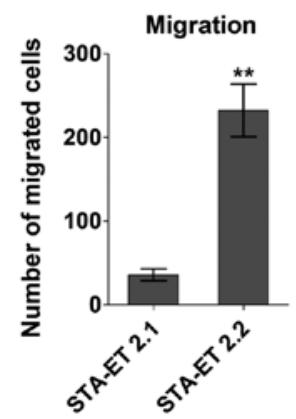

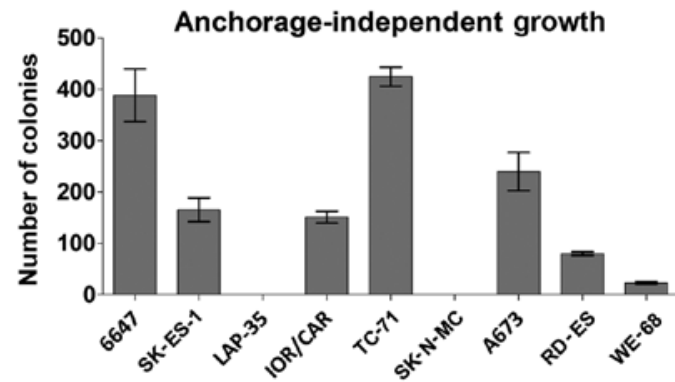

Anchorage-independent

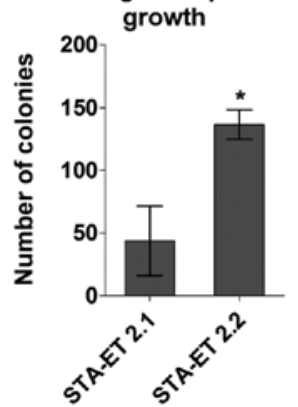

Figure 1. ROCK2, rather than ROCK1, affects Ewing sarcoma (EWS) malignancy. (A) Densitometric analysis of ROCK1 and ROCK2 expression evaluated by western blotting in a representative panel of EWS cell lines. ROCK1/actin and ROCK2/actin ratios are expressed as adjusted volume optical density (OD/mm $\left.{ }^{2}\right)$ and each column represents mean \pm SE of at least three separate experiments. (B) Migration ability of EWS cell lines. Each column represents mean \pm SE of at least two separate experiments. (C) Anchorage-independent growth of EWS cell lines. Each column represents mean \pm SE of at least two separate experiments. (D) Western blotting of ROCK2 and ROCK1 expression in STA-ET 2.1 and STA-ET 2.2 EWS cell lines. Equal loading was monitored by anti-actin blotting. (E) Migration ability (left panel) and anchorage-independent growth (right panel) of the STA-ET 2.1 and STA-ET 2.2 EWS cell lines. Each column represents mean \pm SE of at least two separate experiments. $\mathrm{P} \leq 0.05 ;{ }^{* *} \mathrm{P} \leq 0.001$, paired Student's t-test.

Mebcyto ${ }^{\circledR}$ apoptosis kit; Medical \& Biological Laboratories, Naka-ku Nagoya, Japan).

Statistical analysis. Correlation analysis was performed using Spearman's rank correlation coefficient. Differences among means were analyzed using the 2-tailed Student's t-test.

\section{Results}

ROCK2, rather than ROCK1, affects EWS malignancy. Taking into account the pivotal role of ROCK-kinases in regulating actin cytoskeleton and cell movement, ROCK1 and ROCK2 expression levels were evaluated by western blotting on a panel of representative, patient-derived EWS cell lines (Fig. 1A). With the only exception of LAP-35, which barely expressed both the kinases, ROCK1 and ROCK2 were expressed in all EWS cell lines, with a generally higher expression of ROCK1 with respect to ROCK2. However, only the expression of ROCK 2 appeared to be important for EWS aggressive beha- vior. Indeed, whenever ROCK expression levels were analyzed in relation to migration capabilities and anchorage-independent growth properties of EWS cells (Fig. 1B and C), a direct correlation was observed for ROCK2 (Spearman correlation test: $r=0.791 ; p=0.002$ and $r=0.661 ; p=0.033$ respectively), but not for ROCK1 (Spearman correlation test: $r=0.400 ; p=0.210$ and $\mathrm{r}=0.273 ; \mathrm{p}=0.425$ respectively). This association was confirmed when the expression of the ROCKs was analyzed in STA-ET 2.1 and STA-ET 2.2, two cell lines that were generated from the primary tumor and a bone marrow infiltrate of the same EWS patient (19). Expression of ROCK2, but not of ROCK1, was increased in the cell line that was derived from metastasis compared to that derived from the primary tumor (Fig. 1D), in agreement with the more aggressive phenotype of the STA-ET 2.2 cells (Fig. 1E).

To further confirm the prevalent role of the isoform ROCK 2 in the malignancy of EWS cells, we compared the in vitro efficacy of Stemolecule ${ }^{\mathrm{TM}}$ ROCKII Inhibitor, a specific ROCK2 inhibitor (21) with that of Y27632, which blocks both 
A



B

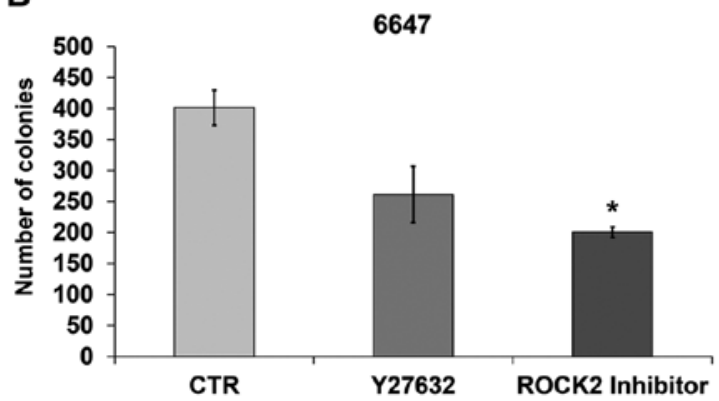

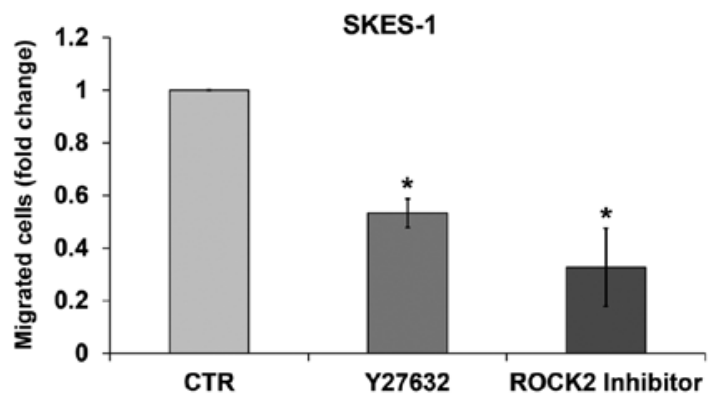

SKES-1

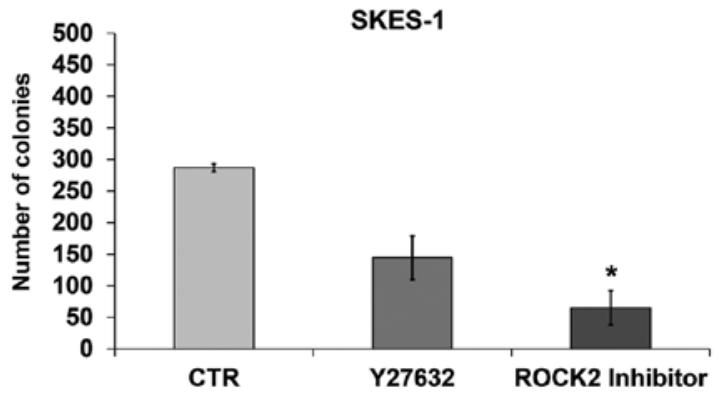

Figure 2. ROCK2 inhibition impairs the migratory properties and anchorage-independent growth capabilities of human Ewing sarcoma (EWS) cell lines. Effect of the Y27632 inhibitor $(10 \mu \mathrm{M})$ and the Stemolecule ${ }^{\mathrm{TM}}$ ROCKII Inhibitor $(10 \mu \mathrm{M})$ on (A) migration and (B) growth in soft agar of 6647 (left panels) and SKES-1 (right panels) cell lines. Each column represents the mean \pm SE of at least two separate experiments performed in triplicate. ${ }^{*} \mathrm{P} \leq 0.05 ;^{* *} \mathrm{P} \leq 0.001$, paired Student's t-test.

ROCK1 and ROCK2 activity. Activity of the two compounds was analyzed in the 6647 and SKES-1 cell lines, as representative of EWS cells with a high or intermediate expression of ROCK2. Both inhibitors significantly reduced the migration of the EWS cells in vitro. The specific ROCK2 inhibitor appeared, however, to be more effective, particularly in the cells showing the highest expression of ROCK2 (Fig. 2A). The higher efficacy of the ROCK2 inhibitor was also confirmed with respect to cell growth in an anchorage-independent condition. The number of colonies in soft agar, an in vitro assay closely suggestive of tumor malignancy level (22), was significantly lower after cell exposure to the ROCK2 inhibitor than to Y27632 (Fig. 2B), further confirming the prevalent role of ROCK2 in regulating EWS aggressiveness.

Blockage of ROCK2 activity inhibits cell proliferation and favors cell differentiation of EWS cells. EWS, 6647 and SKES-1 cells were treated with the ROCK 2 or Y27632 inhibitor in monolayer conditions to explore the additional effects of these agents on cell proliferation, survival and differentiation. Recent reports have shown that the RhoA-ROCK pathway is pivotal in the control of neurite outgrowth and its inhibition (23). We showed here that inhibition of ROCK2 improved neuronal differentiation of EWS cells. Both Y27632 and the specific ROCK2 inhibitor were able to promote neurite outgrowth and to induce expression of $\beta$-III-tubulin (Fig. 3A and B). This was accomplished with marked changes in EWS cell shape (Fig. 3C), in line with the role of ROCK as a regulator of cytoskeletal dynamics: EWS cells lost the capability to grow in suspension, acquired increased adherence to the culture matrix and developed long neurite-like extensions, acquiring a cellular phenotype consistent with neural differentiation. Although these effects were observed after exposure of the cells to the two inhibitors, ROCK2 inhibitor appeared to be more effective in inducing cell shape variations and neural differentiation already at the lower dose of $2 \mu \mathrm{M}$ (Fig. 3B and C). When tumor growth was examined in parallel with differentiation, we observed reduction in the EWS cell growth rate only after treatment with the ROCK2 inhibitor but not with the pan-ROCK inhibitor Y27632 (Fig. 4). Neither ROCK2 inhibitor nor Y27632 induced apoptotic cell death (data not shown).

\section{Discussion}

In the present study, we provide evidence that the specific blockage of ROCK2 activity significantly affects cell migration and growth, and induces marked modification in cell shape, driving EWS cells toward a neurally differentiated phenotype. The role of morphology in tumor progression has become evident in recent years and the shape of a cell has been shown to regulate cancer cell differentiation, proliferation, survival, and motility through the activation of mechanosensitive transcriptional regulators, which are able to link shape information with gene expression (23-30). Despite the existing evidence on the role of the transcriptional coactivators Yes-associated protein (YAP) and transcriptional coactivator with PDZ-binding motif (TAZ) in mechanotransduction, the signaling mediators linking cell shape to modulation of cell differentiation and migration are still poorly understood. ROCK kinases have been shown to participate in a wide range of cellular functions, including control of cell morphology, proliferation and differentiation in addition to their well-known role as regulators of cell migration and invasion (31). These effects are likely due to ROCK activity in the regulation of cytoskeletal structures and the formation of actin stress fibers. Noteworthy, YAP/TAZ response to 
A

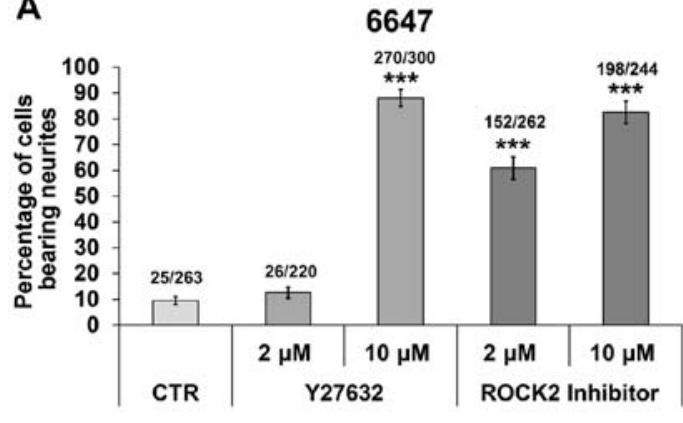

SKES-1



B
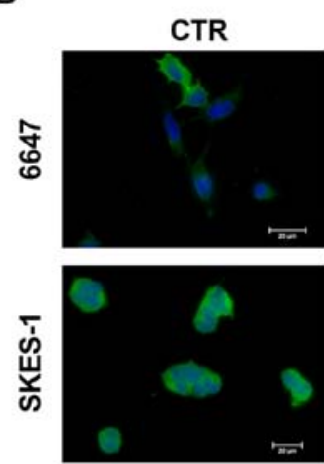

C
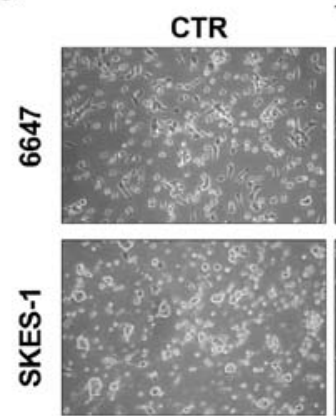

Y27632
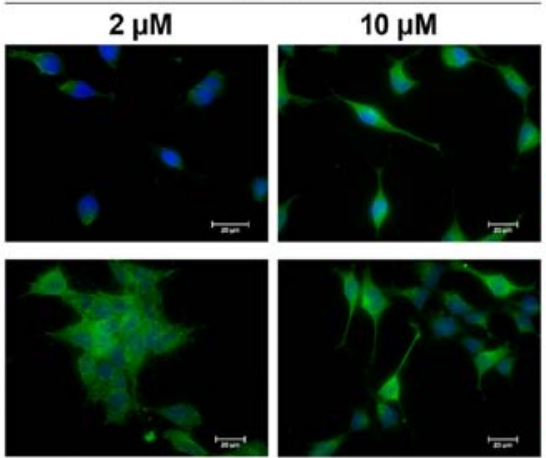

Y27632



$10 \mu \mathrm{M}$
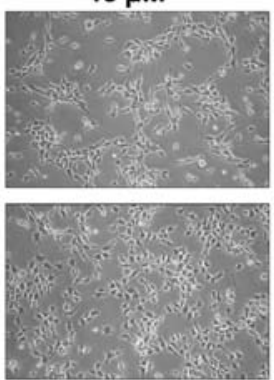

ROCK2 Inhibitor
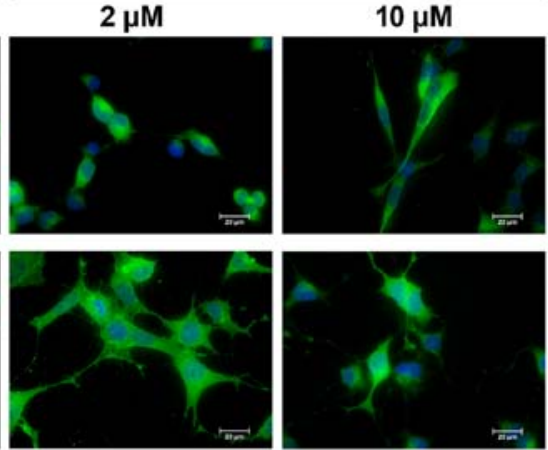

Figure 3. ROCK2 inhibition affects the differentiation properties of Ewing sarcoma (EWS) cells. (A) Effect of the Y27632 inhibitor $(2 \mu \mathrm{M}$ and $10 \mu \mathrm{M})$ and the Stemolecule ${ }^{\mathrm{TM}}$ ROCKII Inhibitor $(2 \mu \mathrm{M}$ and $10 \mu \mathrm{M})$ on neurite outgrowth. Each column represents the mean $\pm \mathrm{SE}$ of the percentage of cells bearing neurites from at least five randomly selected fields. The numbers above each bar represent the ratio between the total number of cells bearing neurites and the total number of cells counted. ${ }^{* * *} \mathrm{P} \leq 0.0001$, paired Student's t-test. (B) Immunostaining of $\beta$ III-tubulin in the 6647 (upper panel) and SKES-1 cells (lower panel) treated with Y27632 inhibitor $(2 \mu \mathrm{M}$ and $10 \mu \mathrm{M})$ or the ROCK2 inhibitor $(2 \mu \mathrm{M}$ and $10 \mu \mathrm{M})$. Digital images were taken in identical conditions using the image analysis software NIS-Elements (Nikon Instruments S.p.A.). Magnification, x600; scale bar, $20 \mu \mathrm{m}$. (C) Effect of the Y27632 inhibitor (2 $\mu \mathrm{M}$ and $10 \mu \mathrm{M})$ and the ROCK2 inhibitor $(2 \mu \mathrm{M}$ and $10 \mu \mathrm{M})$ on 6647 (upper panel) and SKES-1 (lower panel) cell morphology. Magnification, x100.
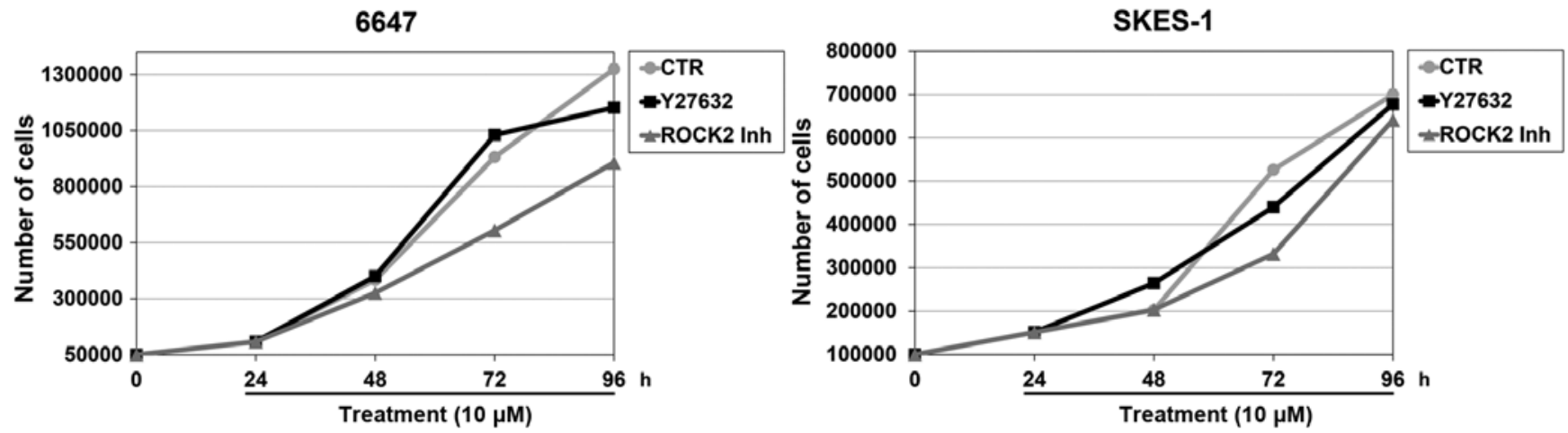

Figure 4. ROCK2 inhibition and proliferation of Ewing sarcoma (EWS) cells. Effect of the Y27632 inhibitor $(10 \mu \mathrm{M})$ and the ROCK2 inhibitor (10 $\mu \mathrm{M})$ on the growth in monolayer cell culture of 6647 and SKES-1 cell lines. 
cytoskeletal tensions requires Rho/ROCK signaling, and inhibition of ROCK2 significantly inhibits nuclear localization of YAP/TAZ (25). In this study, we highlighted the privileged role of ROCK2 with respect to ROCK1 in the regulation of EWS malignancy. It is the specific inhibition of ROCK2, rather than the use of a pan-ROCK inhibitor, that significantly reduced cell migration and growth in vitro, while inducing cell differentiation. This evidence is in line with our previous study concerning the role of ROCK2 in osteosarcoma (21). The effects of ROCK 2 inhibition on cell differentiation may be particularly relevant in mesenchymal tumors, which are highly malignant and poorly differentiated cancers. Differently from other solid tumors, sarcomas are thought to derive from molecular aberrations occurring during the differentiation process of mesenchymal stem cells and they could be reprogrammed to resume normal differentiation (32). The specific inhibition of ROCK2 thus offers an intriguing approach for the design of new options for the treatment of these tumors, also considering that any effects on cell differentiation in sarcomas may also affect stem cell pluripotency and cell fate. We found that, in EWS cells, ROCK2 inhibition induced the acquisition of a neuronlike morphology, increased expression of neuronal markers and, concurrently, slowed down proliferation. This is in line with studies that highlight the pivotal role of the RhoA-ROCK pathway in the control of neurite outgrowth (23), and the pro-differentiation activity of ROCK inhibitors in mesenchymal stem cells towards the neuronal lineage (33). Achieving a greater understanding of the pathways involved in neuritogenesis may help the identification of novel therapeutic targets for the treatment of neurodegenerative diseases. By indicating for the first time that ROCK2, besides its well-described anti-migratory activity, is also crucial for re-directing a tumor cell toward neural differentiation, the present study opens new avenues for the therapeutic use of ROCK inhibitors also in oncology.

Overall, our observations indicate that the specific inhibition of ROCK2 can facilitate EWS cell differentiation toward a neural phenotype, in addition to decreasing cell proliferation and migration. Although all of these effects were also observed by using the pan-ROCK inhibitor Y27632, specific deprivation of ROCK2 activity provided an advantage in terms of efficacy. Further investigations in term of toxicity, dosage and side effects are warranted. However our findings render ROCK2 inhibition a promising candidate for novel treatment against EWS.

\section{Acknowledgements}

The authors would like to thank C. Ghinelli and E.E. Pinca for their help in editing the manuscript. This study was supported by grants from the Associazione Italiana per la Ricerca sul Cancro (AIRC; IG14049 to K.S.), the Liddy Shriver Sarcoma Initiative (international grant to K.S.) and Ricerca Fondamentale Orientata (RFO 2012 to C.Z.). R.S.P. received a fellowship from the Associazione Onlus 'il Pensatore: Matteo Amitrano' and 'Liberi di Vivere Luca Righi'.

\section{References}

1. Bernstein M, Kovar H, Paulussen M, Randall RL, Schuck A, Teot LA and Juergens H: Ewing's sarcoma family of tumors: Current management. Oncologist 11: 503-519, 2006.

2. Riggi N and Stamenkovic I: The biology of Ewing sarcoma. Cancer Lett 254: 1-10, 2007.
3. Campanacci M: Ewing's sarcoma, primitive neuroectodermal tumor (PNET). In: Bone and Soft Tissue Tumors. 2nd edition Springer-Verlag Wien, Vienna, pp653-682, 1999.

4. Paulussen M, Craft AW, Lewis I, Hackshaw A, Douglas C, Dunst J, Schuck A, Winkelmann W, Köhler G, Poremba C, et al; European Intergroup Cooperative Ewing's Sarcoma Study-92: Results of the EICESS-92 Study: Two randomized trials of Ewing's sarcoma treatment - cyclophosphamide compared with ifosfamide in standard-risk patients and assessment of benefit of etoposide added to standard treatment in high-risk patients. J Clin Oncol 26: 4385-4393, 2008.

5. Luksch R, Tienghi A, Hall KS, Fagioli F, Picci P, Barbieri E, Gandola L, Eriksson M, Ruggieri P, Daolio P, et al: Primary metastatic Ewing's family tumors: Results of the Italian Sarcoma Group and Scandinavian Sarcoma Group ISG/SSG IV Study including myeloablative chemotherapy and total-lung irradiation. Ann Oncol 23: 2970-2976, 2012.

6. Amano M, Nakayama M and Kaibuchi K: Rho-kinase/ROCK: A key regulator of the cy toskeleton and cell polarity. Cytoskeleton 67: 545-554, 2010.

7. Wong CCL, Wong CM, Tung EKK, Man K and Ng IOL: Rho-kinase 2 is frequently overexpressed in hepatocellular carcinoma and involved in tumor invasion. Hepatology 49: 1583-1594, 2009.

8. Liu X, Choy E, Hornicek FJ, Yang S, Yang C, Harmon D, Mankin $\mathrm{H}$ and Duan Z: ROCK1 as a potential therapeutic target in osteosarcoma. J Orthop Res 29: 1259-1266, 2011.

9. Lane J, Martin TA, Watkins G, Mansel RE and Jiang WG: The expression and prognostic value of ROCK I and ROCK II and their role in human breast cancer. Int J Oncol 33: 585-593, 2008.

10. Kamai T, Yamanishi T, Shirataki H, Takagi K, Asami H, Ito Y and Yoshida K: Overexpression of RhoA, Rac1, and Cdc42 GTPases is associated with progression in testicular cancer. Clin Cancer Res 10: 4799-4805, 2004.

11. Vishnubhotla R, Sun S, Huq J, Bulic M, Ramesh A, Guzman G, Cho M and Glover SC: ROCK-II mediates colon cancer invasion via regulation of MMP-2 and MMP-13 at the site of invadopodia as revealed by multiphoton imaging. Lab Invest 87: 1149-1158, 2007.

12. Kamai T, Tsujii T, Arai K, Takagi K, Asami H, Ito $\mathrm{Y}$ and Oshima H: Significant association of Rho/ROCK pathway with invasion and metastasis of bladder cancer. Clin Cancer Res 9: 2632-2641, 2003.

13. Zhang C, Zhang S, Zhang Z, He J, Xu Y and Liu S: ROCK has a crucial role in regulating prostate tumor growth through interaction with c-Myc. Oncogene 33: 5582-5591, 2014.

14. Wang N, Feng Y, Lau EP, Tsang C, Ching Y, Man K, Tong Y, Nagamatsu T, Su W and Tsao S: F-Actin reorganization and inactivation of Rho signaling pathway involved in the inhibitory effect of Coptidis Rhizoma on hepatoma cell migration. Integr Cancer Ther 9: 354-364, 2010.

15. Patel RA, Liu Y, Wang B, Li R and Sebti SM: Identification of novel ROCK inhibitors with anti-migratory and anti-invasive activities. Oncogene 33: 550-555, 2014.

16. Yoneda A, Multhaupt HA and Couchman JR: The Rho kinases I and II regulate different aspects of myosin II activity. J Cell Biol 170: 443-453, 2005.

17. Yoneda A, Ushakov D, Multhaupt HAB and Couchman JR: Fibronectin matrix assembly requires distinct contributions from Rho kinases I and -II. Mol Biol Cell 18: 66-75, 2007.

18. Mertsch S and Thanos S: Opposing signaling of ROCK1 and ROCK 2 determines the switching of substrate specificity and the mode of migration of glioblastoma cells. Mol Neurobiol 49: 900-915, 2014.

19. Kovar H, Pospisilova S, Jug G, Printz D and Gadner H: Response of Ewing tumor cells to forced and activated p53 expression. Oncogene 22: 3193-3204, 2003.

20. Bagnara GP, Serra M, Giovannini M, Badiali M, Stella M, Montaldi A, Granchi D, Paolucci P, Rocchi P, Pession A, et al: Establishment and characterization of a primitive neuroectodermal tumor of bone continuous cell line (LAP-35). Int J Cell Cloning 8: 409-424, 1990.

21. Zucchini C, Manara MC, Pinca RS, De Sanctis P, Guerzoni C, Sciandra M, Lollini PL, Cenacchi G, Picci P, Valvassori L, et al: CD99 suppresses osteosarcoma cell migration through inhibition of ROCK2 activity. Oncogene 33: 1912-1921, 2014.

22. Manara MC, Bernard G, Lollini PL, Nanni P, Zuntini M, Landuzzi L, Benini S, Lattanzi G, Sciandra M, Serra M, et al: CD99 acts as an oncosuppressor in osteosarcoma. Mol Biol Cell 17: 1910-1921, 2006. 
23. Yang P, Wen HZ and Zhang JH: Expression of a dominant-negative Rho-kinase promotes neurite outgrowth in a microenvironment mimicking injured central nervous system. Acta Pharmacol Sin 31: 531-539, 2010.

24. Cordenonsi M, Zanconato F, Azzolin L, Forcato M, Rosato A, Frasson C, Inui M, Montagner M, Parenti AR, Poletti A, et al: The Hippo transducer TAZ confers cancer stem cell-related traits on breast cancer cells. Cell 147: 759-772, 2011.

25. Dupont S, Morsut L, Aragona M,Enzo E, GiulittiS, Cordenonsi M, Zanconato F, Le Digabel J, Forcato M, Bicciato S, et al: Role of YAP/TAZ in mechanotransduction. Nature 474: 179-183, 2011.

26. Aragona M, Panciera T, Manfrin A, Giulitti S, Michielin F, Elvassore N, Dupont S and Piccolo S: A mechanical checkpoint controls multicellular growth through YAP/TAZ regulation by actin-processing factors. Cell 154: 1047-1059, 2013.

27. Chen CS, Mrksich M, Huang S, Whitesides GM and Ingber DE: Geometric control of cell life and death. Science 276: 1425-1428, 2008.

28. Sero JE, Thodeti CK, Mammoto A, Bakal C, Thomas S and Ingber DE: Paxillin mediates sensing of physical cues and regulates directional cell motility by controlling lamellipodia positioning. PLoS One 6: e28303. 2011.
29. Mcbeath R, Pirone DM, Nelson CM, Bhadriraju K and Chen CS: Cell shape, cytoskeletal tension, and RhoA regulate stem cell lineage commitment. Dev Cell 6: 483-495, 2004.

30. Discher DE, Mooney DJ and Zandstra PW. Growth factors, matrices, and forces combine and control stem cells. Science 324: 1673-1677, 2009.

31. Julian L and Olson MF: Rho-associated coiled-coil containing kinases (ROCK): Structure, regulation, and functions. Small GTPases 5: e29846, 2014.

32. Charytonowicz E, Terry M, Coakley K, Telis L, Remotti F, Cordon-Cardo C, Taub RN and Matushansky I: PPAR $\gamma$ agonists enhance ET-743-induced adipogenic differentiation in a transgenic mouse model of myxoid round cell liposarcoma. J Clin Invest 122: 886-898, 2012.

33. Liu X, Zhang Z, Yan X, Liu H, Zhang L, Yao A, Guo C, Liu X, $\mathrm{Xu}$ T: The Rho kinase inhibitor Y-27632 facilitates the differentiation of bone marrow mesenchymal stem cells. J Mol Histol 45: 707-714, 2014. 